\title{
A Short Review on Phytoconstituents from Genus Albizzia and Erythrina
}

\author{
Mohammad Musarraf Hussain \\ Department of Pharmacy, Faculty of Life and Earth Sciences, Jagannath University, \\ Dhaka-1100, Bangladesh
}

(Received: 20 May, 2018; Accepted: 18 July, 2018; Published: 31 July, 2018)

\begin{abstract}
The genus Albizzia and Erythrinaare the leading sources of phytoconstituents. The aim of this review is to solicitude of the phytoconstituents from some medicinal plants of these genus. A total nine medicinal plants were studied and 121 chemical constituents along with structures have been reported here. Erythrina burttii consists of highest number of constituents.
\end{abstract}

Key words: Medicinal plant, phytoconstituents, flavonoids, isoflavanones, saponins, sesquiterpenes, flavan, steroid, triterpenoids.

\section{Introduction}

Nature is a great source of medicinal plants and these plants are used as a traditional medicine for many years (Hussain et al., 2010). One hundred and ten species are present as trees and shrubs in the genus Erythrina (Hussain et al., 2016a, 2011). Among them, two species have been syudied comprehensively in this review. E. burttii is a flowering and flat-topped tree (height: $3.5-18 \mathrm{~m}$ ) growing in Ethiopia, Kenya and Tanzania.E. droogmansiana is a single straight stem, soft wood and rounded crown tree (height: $20 \mathrm{~m}$ ) extensively grown in Congo, Cameron and Gabon, and used in the treatment of fever, hemorrhoids, and wound infection in locally. The genus Albizzia consists of 150 species extensively distributed in Africa, Asia, and South America. Albizzia species were used as traditional medicine in the treatment of anthelmintic, cough, diarrhea, insomnia, irritability, injuries, poor memories, rheumatism, scabies, stomach trouble, and wounds in Africa and China (Hussain et al., 2016b). A. anthelmintica is a medium canopied tree (height: 8 m) with soft bark and unwrap spine. A. lebbeck (Leguminosae) is an exposed deciduous tree (height:
12-21 m) that grows in over Bangladesh (Hussain et al., 2008). A. inundata is a perennial tree and found in Argentina. A. glaberrima is a big tree having few flattened crown and used as a folk medicine in the treatment of anemia, blenorrhagia, bilharzias, epilepsy, and liver complications in Cameroon and Nigeria. A. coriaria is a medicinal plant found in Uganda and used in the treatment of eye diseases, jaundice, skin disease, sore throats, and syphilis. $C$. zeyheri (Family: Combretaceae) is a Tanzanian medicinal plant and applied for the management of different health consequences such as cancer, cough diarrhea, hypertension, and snakebite.

\section{Reported phytoconstituents}

A total nine medicinal plants have been studied and one hundred and twenty one (1-121) compounds were reported in this review as phytoconstituents. The studied medicinal plants are Erythrina burttii, E. droogmansiana, Albizzia submidiata, $A$. anthelmintica, A. inundata, Spergularia marginata, Manikara rufula, A. lebbeck, Ainsliaea yunnanenssi, A. glaberrima, Combretum zeyheri, A. boromoensis, A. grandibracteata, and A. coriaria.

Correspondence to: Mohammad Musarraf Hussain, Email: m.musarraf.hussain@gmail.com 


\section{Flavonoids}

Erythrina genus is a pioneer source of flavonoids. There are twenty four phytoconstituents have been reported as flavonoids and pilocarpin from Erythrina burttii such as Burttinol A (1), Burttinol-A diacetate (2), Burttinol B (3), Burttinol-B acetate (4), Burttinol C (5), Burttinol-C diacetate (6), Eryvarin $\mathrm{H}$ (7), Eryvarin-H diaceate (8), Burttinol D (9),
Burttinol-D diacetae (10), 4- $\alpha$-O-methylsigmoidin B (11), Abyssinone V (12), Abyssinone V methyl ether (13), Calopocarpin (14), Burttinne (15), Neurautenol (16), Bidwillon (17), Isobavachalcone (18), Erythrinasinate (19), 7-O-methylluteone (20), Burttinonedehydrate (21), 8-Prenylluteone (22), 3-Omethylcalopocarpin (23), and genistein (24) (Figure 1) (Yenesew et al., 2012, 1998, 2003).
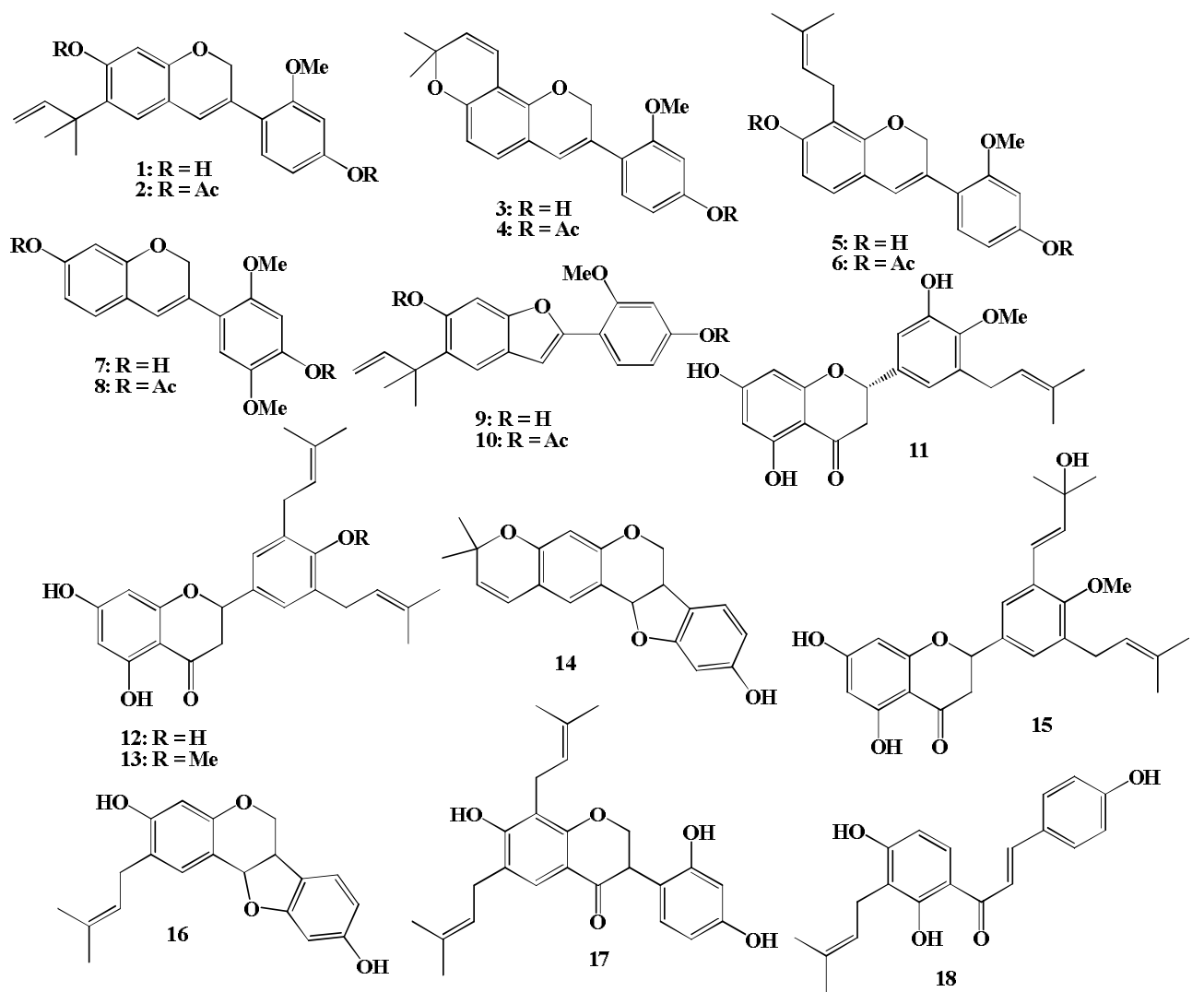<smiles>CCOC(=O)/C=C/c1ccc(O)c(OC)c1</smiles><smiles>COc1cc2c(c(O)c1CC=C(C)C)C(=O)C(C1CC=C(O)C=C1C)=CC2</smiles>

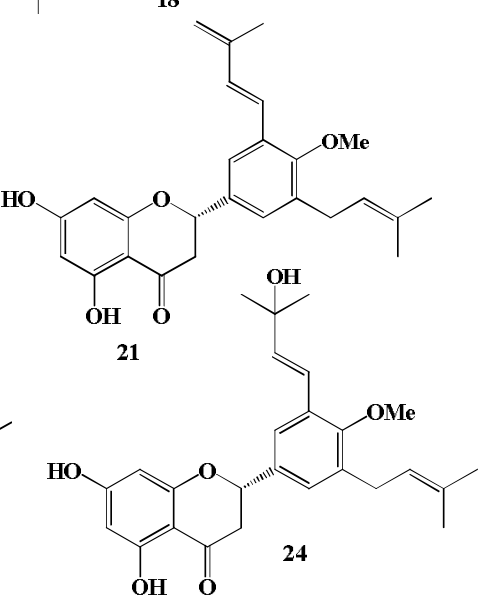

Figure 1. Flavonoids from Erythrina burttii. 


\section{Isoflavanones}

The genus Erythrina (Family: Leguminosae) is a renowned source of isoflavanones and alkaloids. Ten isoflavanones were reported from the root bark of Erythrina droogmansiana for example 7,4'Dihydroxy-2'-methoxy-3'-(3-methylbut-2-enyl)isoflavanone (25), Sophoraisoflavanone A (26),
Erypoegin D (27), Trihydroxy-8-(3-methylbut-2enyl)-[6", $6^{\prime \prime}$-dimethylpyrano $\left.\left(2^{\prime \prime}, 3^{\prime \prime}: 4^{\prime}, 5^{\prime}\right)\right]$ isoflavone (28), Isolupalbigenin (29), 5,7,2',4'-Tertahydroxy8,5'-di-(3-metylbut-2-enyl)isoflavone

(30),

Erypostyrene (31), Phaseollidin (32), Cristacarpin (33), and Erystagallin A (34) (Figure 2) (Bedaneet al., 2017).

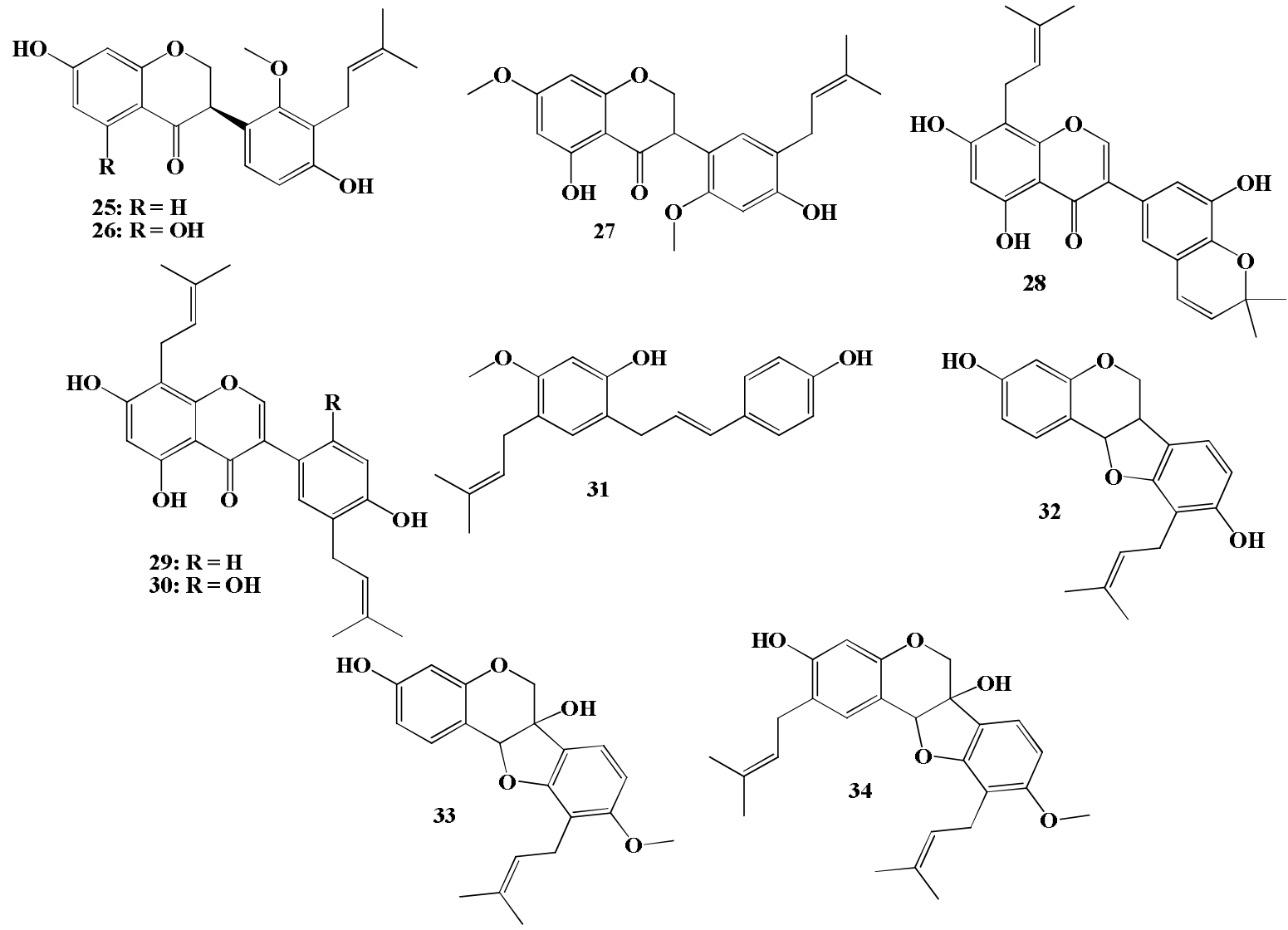

Figure 2. Isoflavanones from Erythrina droogmansiana.

\section{Saponins}

Albizzia subdimidiata, A. anthelmintica, A. inundata, Spergularia marginata, and Manilkara rufula are the key source of saponins. The reported saponins from these plants are 3-O-D-Xylopyranosyl$(1 \rightarrow 2)$-L-arab-inopyranosyl-( $1 \rightarrow 6)$-2-acetamido-2deoxy-D-glucopyranosyl-oleanolic acid (35), 3-O-DXylopyranosyl-( $1 \rightarrow 2)$-L-arabinopyranosyl-( $1 \rightarrow 6)$-2acetamido-2-deoxy-D-glucopyranosyl-oleanolic acid peracetate (36), 3-O-L-Arabinopyranosyl-(1 $\rightarrow 2)-\mathrm{L}-$ arabinopyranosyl-( $1 \rightarrow 6)$-2-acetamido-2-deoxy-Dglucopyranosyl-oleanolic acid (37), 3-O-LArabinopyranosyl-( $1 \rightarrow 2)$-L-arabinopyranosyl$(1 \rightarrow 6)$-2-acetamido-2-deoxy-D-glucopyranosyloleanolic acid peracetate (38), 3-O-LArabinopyranosyl-( $1 \rightarrow 6)$-2-acetamido-2-deoxy-Dglucopyranosyl-oleanolic acid (39), 3-O-2Acetamido-2-deoxy-D-glucopyranosyl-oleanolic acid (40), O-Methyl-cyclitol (41), 3-O- $[\alpha-\mathrm{L}-$ Arabinopyranosyl-( $1 \rightarrow 6)]$-2-acetamido-2-deoxy- $\beta$ - 
D-glucopyranosyl-oleanolic acid (42), 3-O- $[\alpha-\mathrm{L}-$ Arabinopyranosyl-( $(1 \rightarrow 6)]$-2-acetamido-2-deoxy- $\beta$ -

D-glucopyranosyl-echinocystic acid (43), 3-O-[ $\alpha-\mathrm{L}-$ Arabinopyranosyl-( $(1 \rightarrow 2)-\alpha-\mathrm{L}-$ arabinopyranosyl$(1 \rightarrow 6)]$-2-acetamido-2-deoxy- $\beta$-D-glucopyranosylacacic acid lactone (44), 3-O-[ $\beta$-D-xylopyranosyl$(1 \rightarrow 2)-\alpha$-L-arabinopyranosyl-( $1 \rightarrow 6)]$-2-acetamido-2deoxy- $\beta$-D-glucopyranosyl-acacic acid lactone (45), 3-O-[ $\alpha$-L-arabinopyranosyl- $(1 \rightarrow 2)-\alpha$-Larabinopyranosyl-(1 $\rightarrow 6)]-\beta$-D-glucopyranosyloleanolic acid (46), 3-O-[ $\beta$-D-xylopyranosyl- $(1 \rightarrow 2)$ $\alpha$-L-arabinopyranosyl-( $1 \rightarrow 6)]-\beta$-D-glucopyranosyloleanolic acid (47), 3-O-[ $\beta$-D-glucopyranosyl$(1 \rightarrow 2)]-\beta$-D-glucopyranosyl-oleanolic acid (48), 3O-[ $\alpha$-L-arabinopyranosyl- $(1 \rightarrow 2)-\alpha$-Larabinopyranosyl-( $1 \rightarrow 6)$-[ $\beta$-D-glucopyranosyl$(1 \rightarrow 2)]-\beta$-D-glucopyranoside-echinocystic acid (49), 3-O-[ $\beta$-D-xylopyranosyl- $(1 \rightarrow 2)-\alpha-\mathrm{L}-$ arabinopyranosyl- $(1 \rightarrow 6)$-[ $\beta$-D-glucopyranosyl$(1 \rightarrow 2)]-\beta$-D-glucopyranoside-echinocystic acid $(\mathbf{5 0})$, 3 -O-[ $\beta$-D-glucopyranosyl- $(1 \rightarrow 3)]$ - $[\alpha-\mathrm{L}$ arabinopyranosyl- $(1 \rightarrow 2)$-[ $\alpha$-L-arabinopyranosyl$(1 \rightarrow 6)]$-2-acetamido-2-deoxy- $\beta$-D-glucopyranosylechinocystic acid (51) 3-O-[ $\alpha$-L-arabinopyranosyl-
$(1 \rightarrow 2)]$-[ $\alpha$-L-arabinopyranosyl-( $(1 \rightarrow 6)]$-2-acetamido2-deoxy- $\beta$-D-glucopyranosyl-echinocystic acid (52), 3 -O-[ $\alpha$-L-Arabinopyranosyl- $(1 \rightarrow 6)]$-2-acetamido-2deoxy- $\beta$-D-glucopyranosyl-echinocystic acid (53), 3O- $\beta$-D-glucuronopyranosyl-echinocystic acid 28-O$\alpha$-L-arabinopyranosyl-( $1 \rightarrow 2)$ - $\alpha$-L-rhamnopyranosyl$(1 \rightarrow 3)-\beta$-D-xylopyranosyl-( $1 \rightarrow 4)-\alpha-L-$

rhamnopyranosyl- $(1 \rightarrow 2)-\alpha-\mathrm{L}-\quad$ arabinopyranosyl ester (54), 3-O- $\beta$-D-glucopyranosyl- $(1 \rightarrow 3)-\beta$-Dglucuronopyranosyl echinocystic acid 28-O- $\alpha$-Larabinopyranosyl-( $1 \rightarrow 2)-\alpha$-L-rhamnopyranosyl-

$(1 \rightarrow 3)-\beta$-D-xylopyranosyl- $(1 \rightarrow 4)-\alpha-L-$

rhamnopyranosyl-( $(\rightarrow 2)-\alpha$-L-arabinopyranosyl ester (55), 3-O- $\beta$-D-glucopyranosyl- $(1 \rightarrow 4)$-3-O-sulfate- $\beta$ D-glucuronopyranosyl echinocystic acid $28-\mathrm{O}-\alpha-\mathrm{L}-$ arabinopyranosyl-( $1 \rightarrow 2)$ - $\alpha$-L-rhamnopyranosyl-

$(1 \rightarrow 3)-\beta$-D-xylopyranosyl- $(1 \rightarrow 4)-\alpha-L-$

rhamnopyranosyl- $(1 \rightarrow 2)-\alpha$-Larabinopyranosyl ester (56), 3-O- $\beta$-D-glucopyranosyl-( $1 \rightarrow 4)-\beta$-Dglucuronopyranosyl-21-O-acetyl acacic acid (57), and Mi-saponin C (58) (Figure 3) (Kader et al., 2001; Runyoroet al., 2015; Zhanget al., 2011; Carpaniet al., 1989; Pertuitet al., 2017; Vieira et al., 2017).
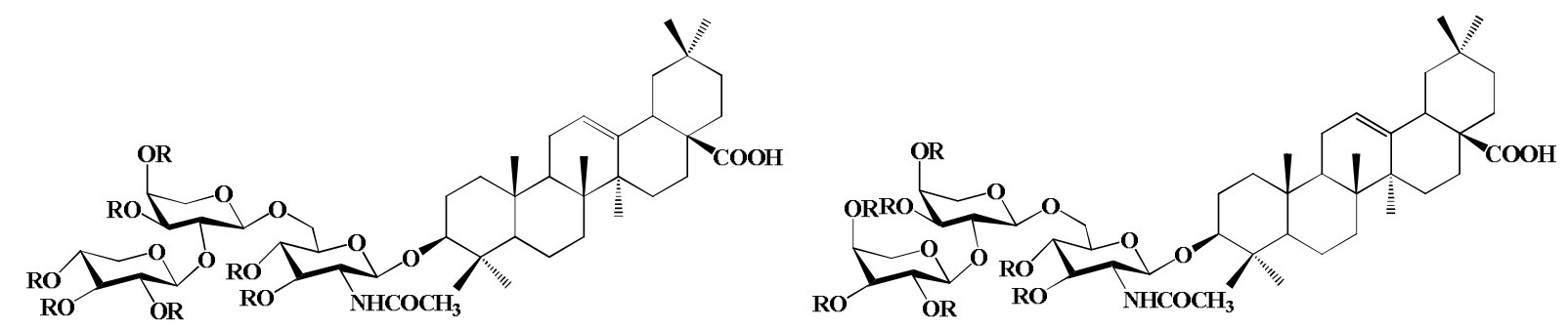

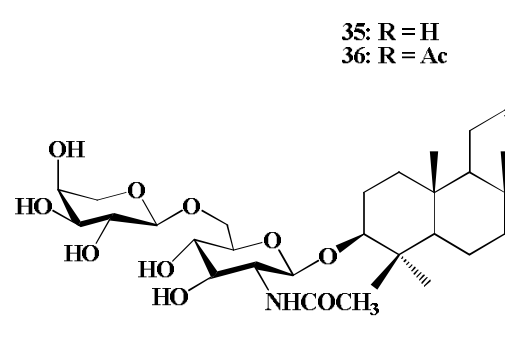

39

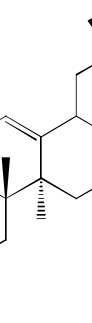

1<smiles>CCCCC(C)(C)O</smiles>

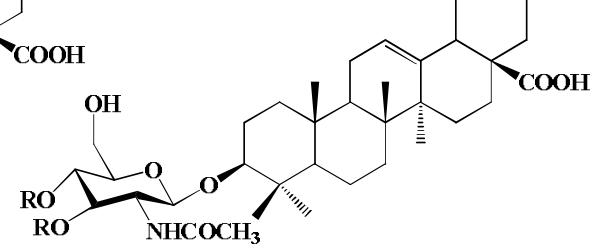

40

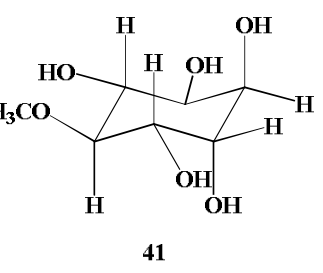

Figure contd. 


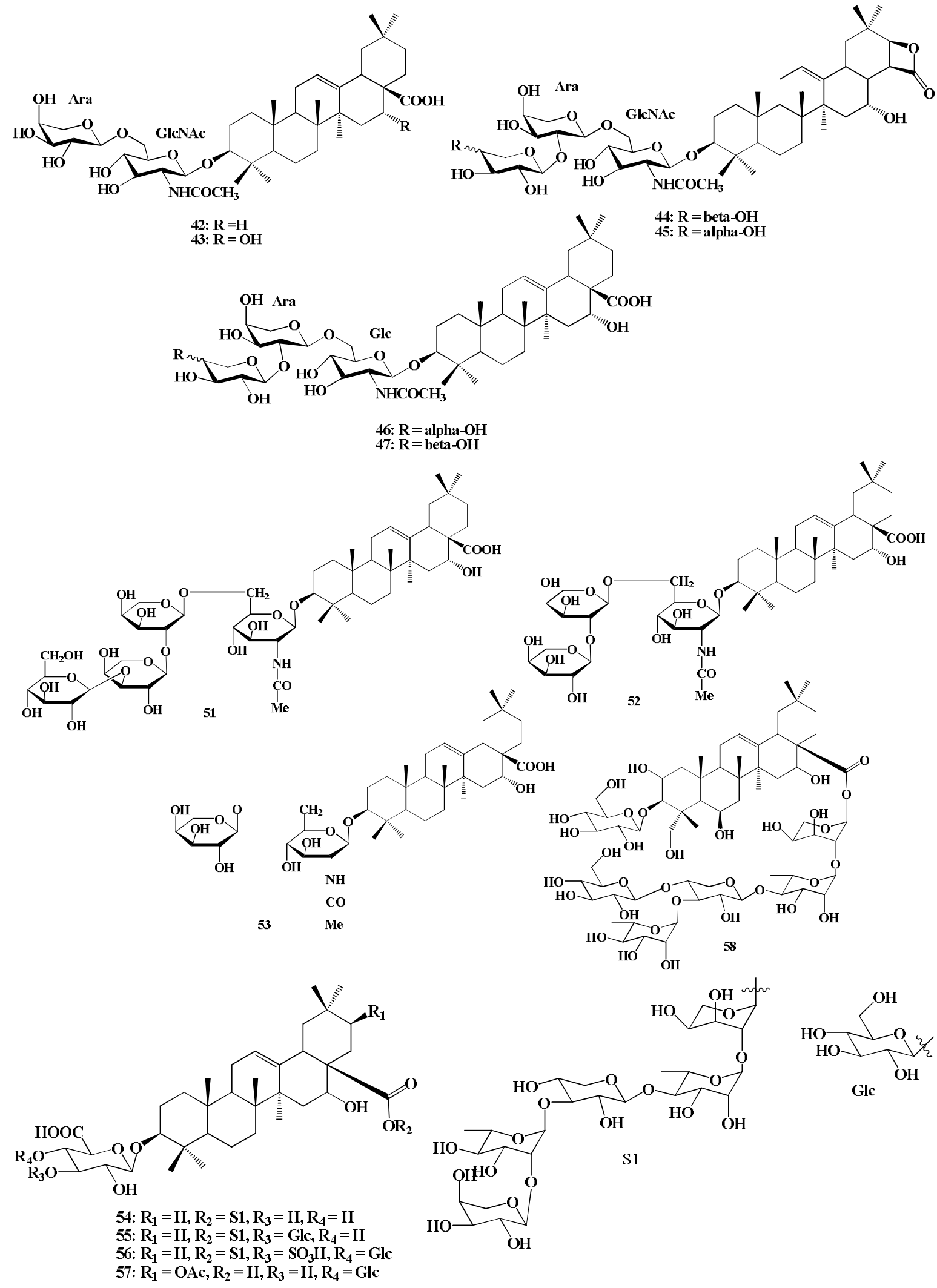

Figure 3. Saponins from medicinal plants. 


\section{Sesquiterpenes}

Aromatic medicinal plant Albizzai lebbeck and Ainsliaea yunnanenssi are the principle source of sesquiterpenes. The elucidated sesquiterpenes from these plant are Benzyl-1-O- $\beta$-D-glucopyranosidem (59),

Benzyl-6-O- $\alpha$-L-arabinopyranosyl- $\beta$-Dglucopyranoside (60), Linalyl- $\beta$-D-glucopyranoside (61), Linalyl-6-O- $\alpha$-L-arabinopyranosyl- $\beta$-Dglucopyranoside (62), (2E)-3,7-Dimethylocta-2,6dienoate-6-O- $\alpha$-L-arabinopyranosyl- $\beta$-D-

glucopyranoside (63), Glycoside1-O-[6-O- $\alpha-\mathrm{L}-$ arabinopyranosyl- $\beta$-D-glucopyranoside]-(2E, 6E-)farnesol (64), $n$-Hexyl- $\alpha$-L arabinopyranosyl- $(1 \rightarrow 6)$ $\beta$-D-glucopyranoside $\quad(65), \quad n$-Octyl- $\alpha$-Larabinopyranosyl-( $1 \rightarrow 6)-\beta$-D-glucopyranoside $\quad(66)$, 2,3-Dihydroxy-2,3-dihydrosqualene (67), Ethyl fructofuranoside (68), Yunnanolides A (69), Yunnanolides B (70), Yunnanolides C (71),
Yunnanolides D (72), Yunnanolides E (73), Yunnanolides F (74), Yunnanolide G (75), Yunnanolides H (76), Yunnanolides I (77), Pertyolide C (78), Diaspanolide A (79), Diaspanolide B (80), 1a-Hydroxy-3-O-isovalerate zaluzanin C (81), Tetrahydrodehydrozaluzanin C (82), Dihydrozaluzanin C (83), Zaluzanin C (84), Isoamberboin (85), 11b,13-Dihydro-3-epizaluznin C (86) , and 4b,15,11b,13-Tetrahydrozaluzanin C (87) (Figures 4 and 5) (Massarani et al., 2016; Fang et al. 2017).

\section{Flavan and Steroids}

A bunch of flavan and steroids isolated from the Albizzia glaberrima such as (+)-(2R,3S,4R)-3',4',7trihydroxy-4-methoxy-2,3-trans-flavan-3,4-trans-diol (88), (+)-Mollisacacidin (89), (+)-Fustin (90), Butin (91), Chondrillasterol (92), and Chondrillasterone (93) (Fotso et al. 2017).

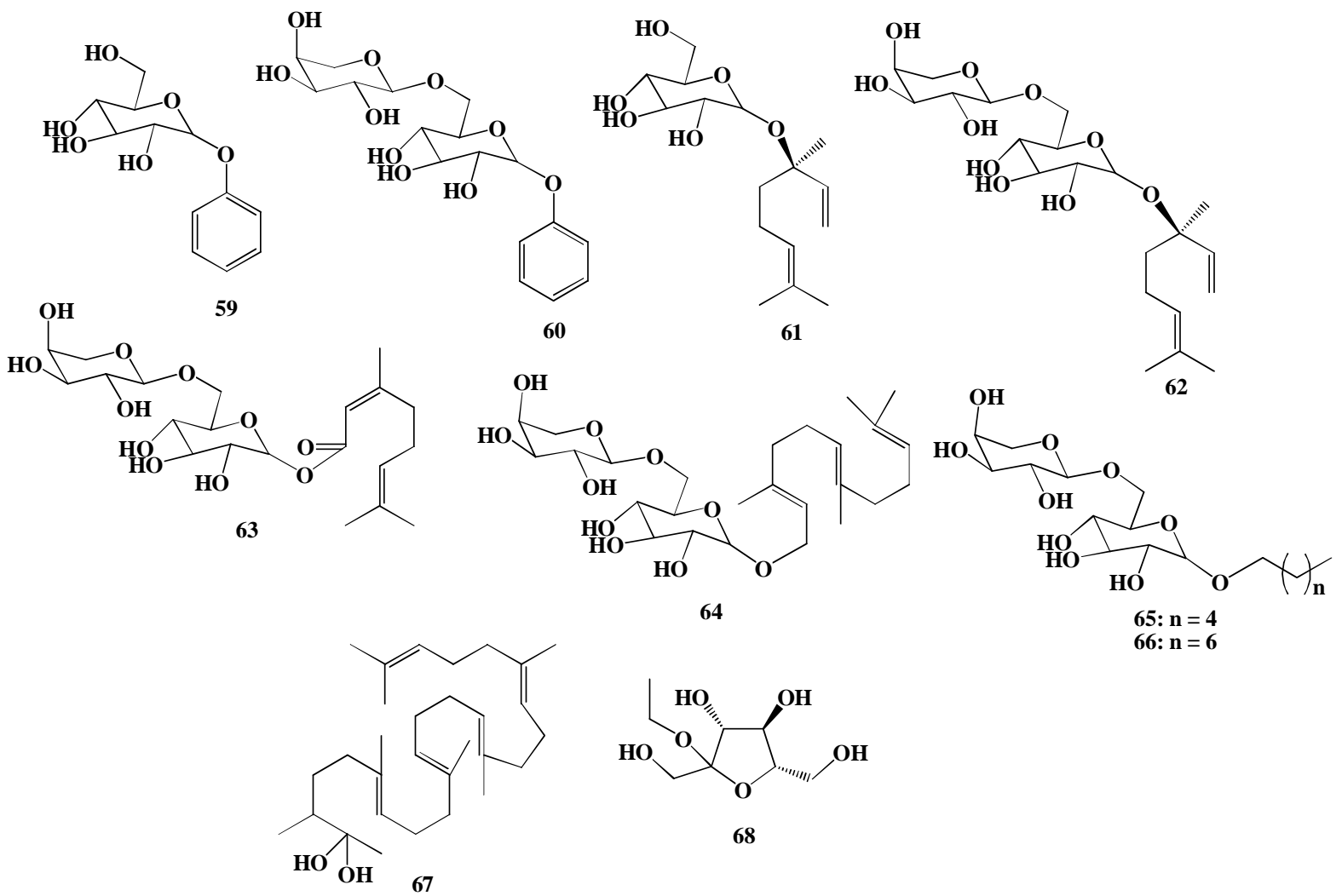

Figure 4. Sesquiterpenes from A. lebbeck. 

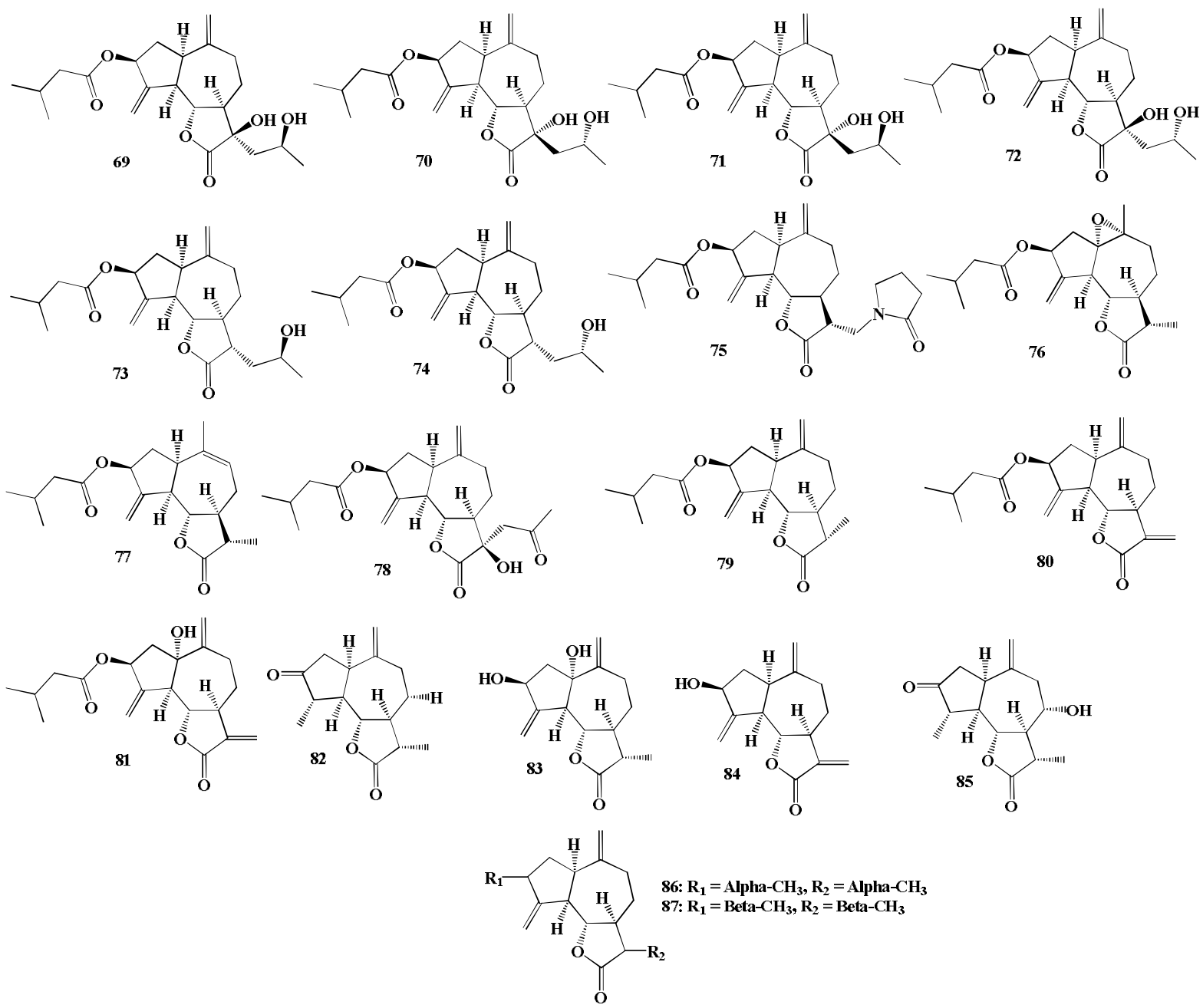

Figure 5. Isolated sesquiterpenes from Ainsliaea yunnanesis.
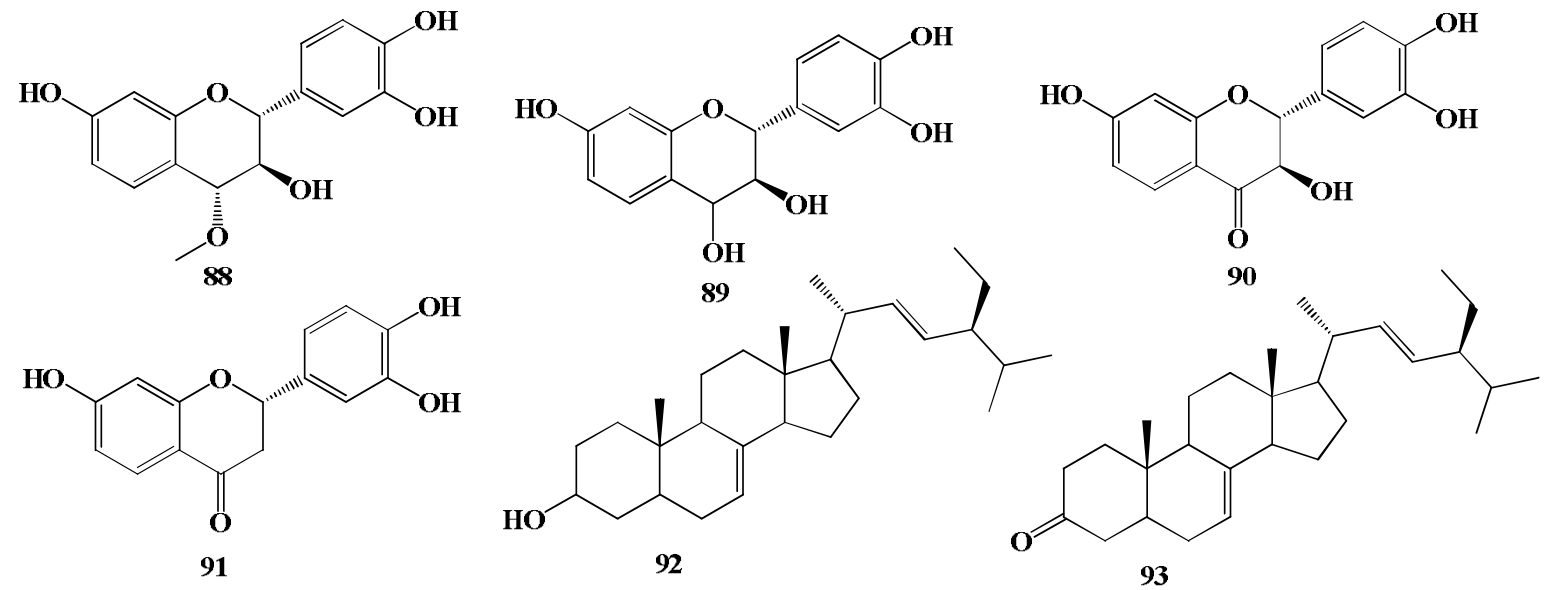

Figure 6. Flavan and Steroids from Albizzia glaberrima. 


\section{Triterpenoids}

A lots of triterpenoids have been derived from Combretum zeyheri, A. glaberrima, A. boromoensis, and A. grandibracteata for example Lupeol (94), Ursolic acid (95), Oleanolic acid (96), Maslinic acid (97), 2 $2 \alpha, 3 \beta$-Dihydroxy-urs-12-en-28-oic acid (98), 6 $\beta$-Hydroxymaslinic acid (99), Terminolic acid (100), Methylsumaresinolate (101), Arjunolic acid (102), Asiatic cid (103), Glaberrimoside A (104),
Glaberrimoside B (105), Glaberrimoside C (106), Boromoenoside A (107), Boromoenoside B (108), Boromoenoside C (109), Boromoenoside D (110), Gummiferaosides D (111), Gummiferaosides E (112), Julibroside $\mathrm{J}_{8}$ (113), Grandibracteoside A (114), Grandibracteoside B (115) and Grandibracteoside C (116) (Runyoro et al., 2013; Note et al., 2016, 2015; Simo et al., 2017; Krief et al., 2005).<smiles>C=C(C)C1CC[C@]2(C)CC[C@]3(C)C(CCC4[C@@]5(C)CC[C@@H](O)[C@](C)(O)C5CC[C@]43C)C12</smiles><smiles>CC1(C)CC[C@@]2(C)C(C1)C1=CCC3[C@](C)(CC[C@@H]12)CC[C@@]1(C(=O)O)CCC(C)(C)[C@@]31C</smiles>

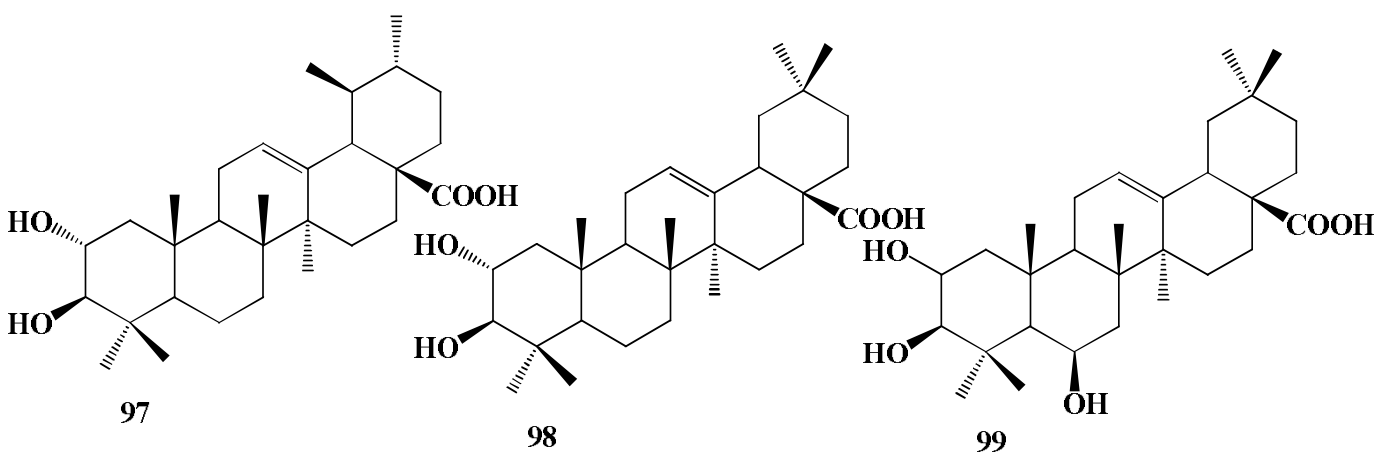

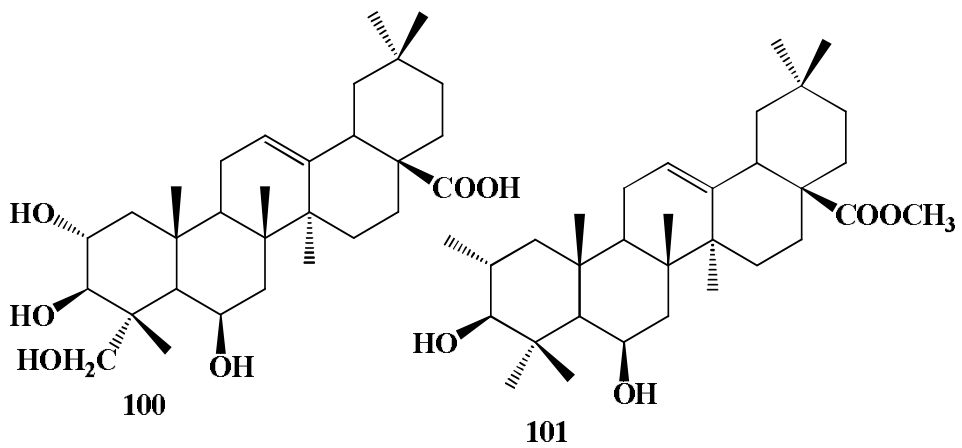

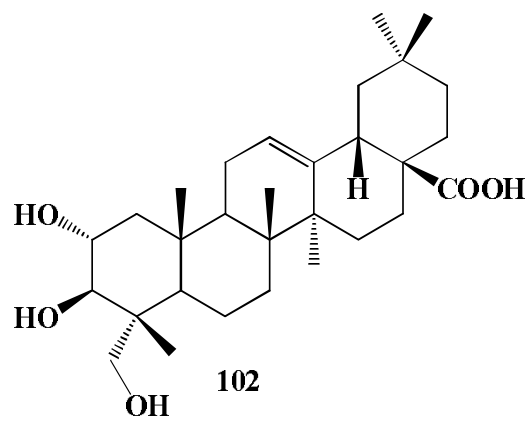



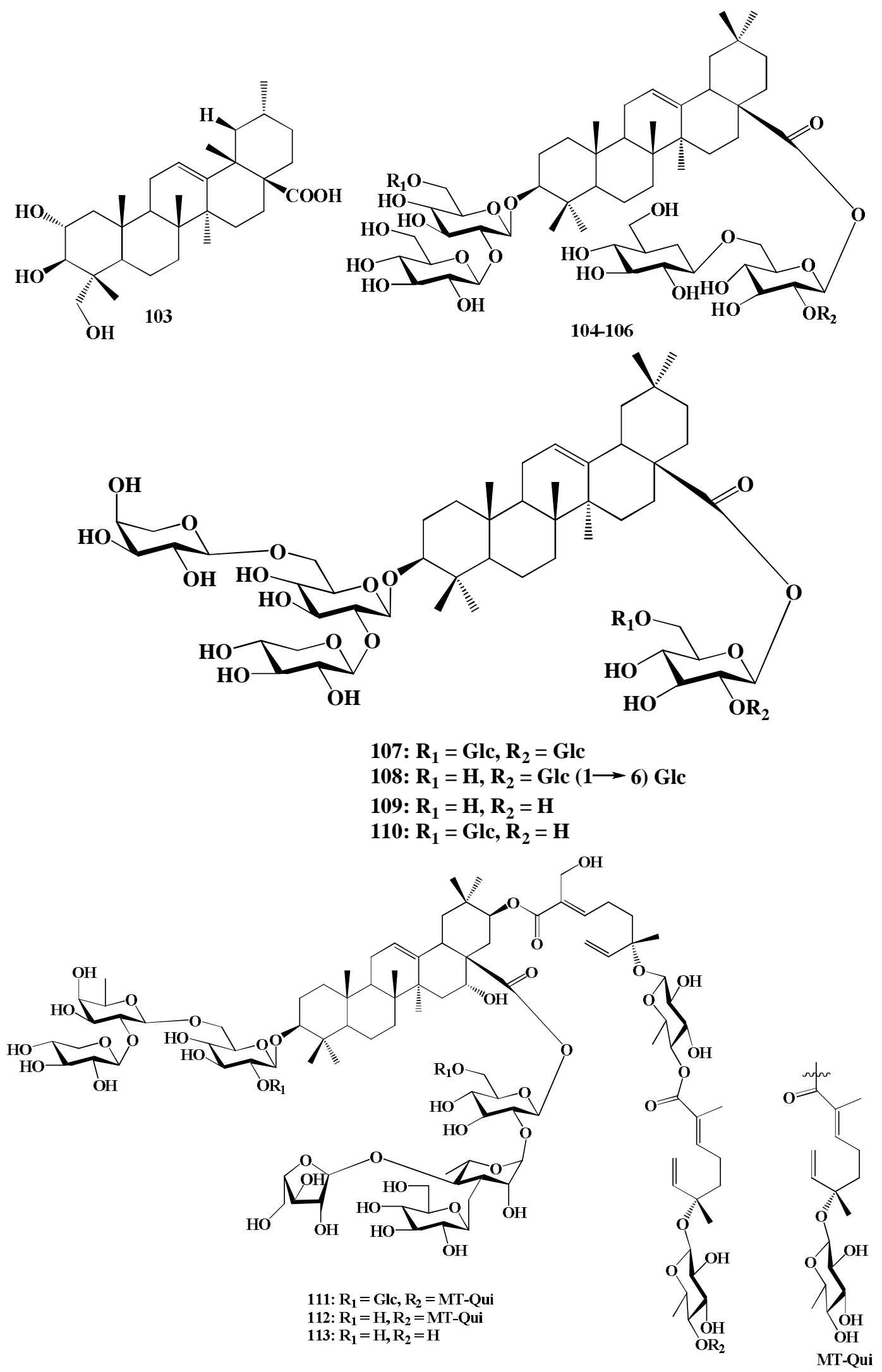

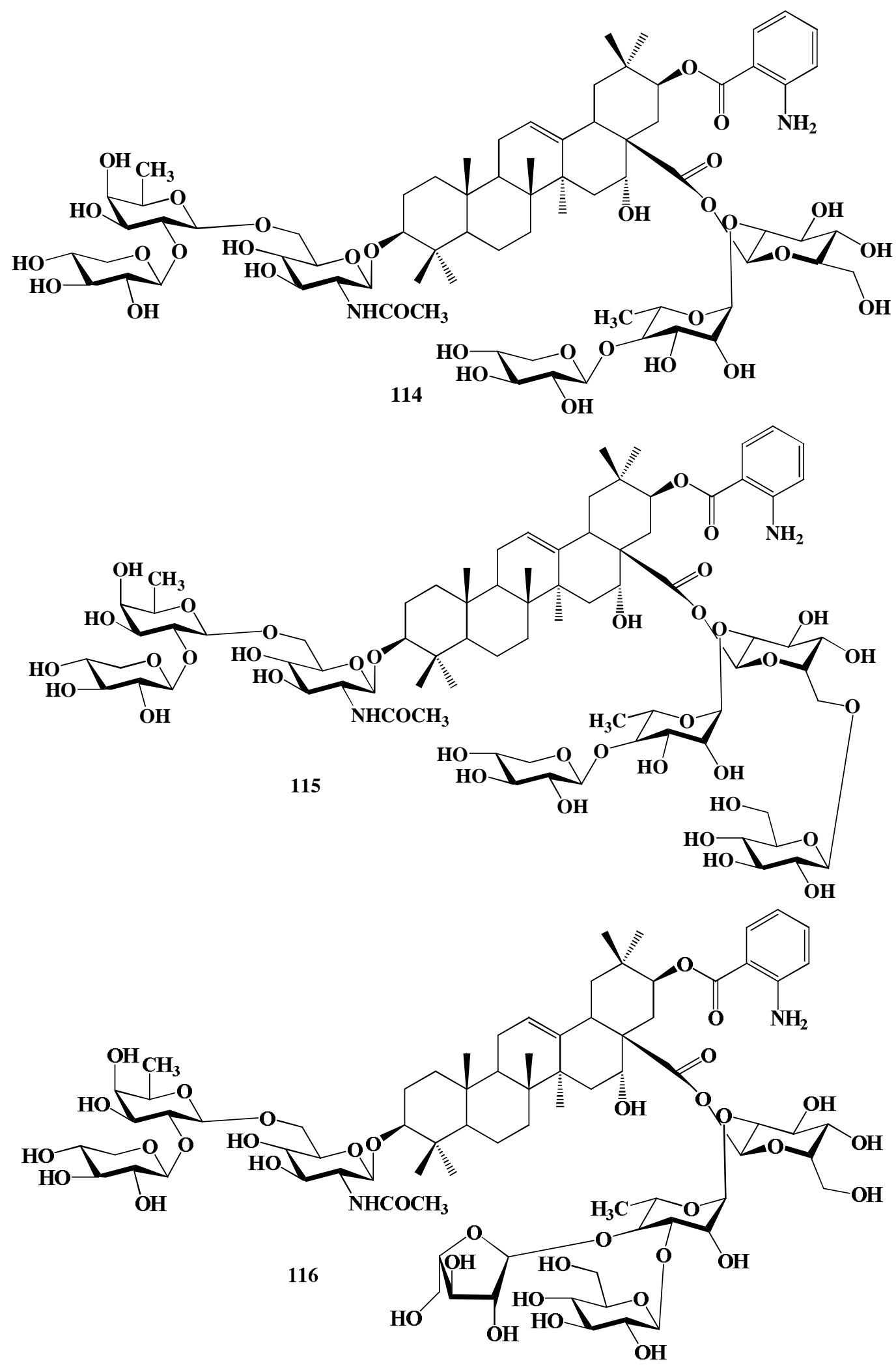

Figure 7. Triterpenoids from different plants. 


\section{Miscellaneous}

A total six molecules for examples Lupeol (94), Lupenone (117), Betulinic acid (118), Acacic acid lactone (119), (+) - Catechin (120), and Benzyl alcohol (121) were isolated with chemical structures from Albizzia coriaria (Figure 7) (Byamukama et al., 2015).

\section{Biological properties}

The reported phytoconstituents showed lots of biological properties that are given in table 1 .

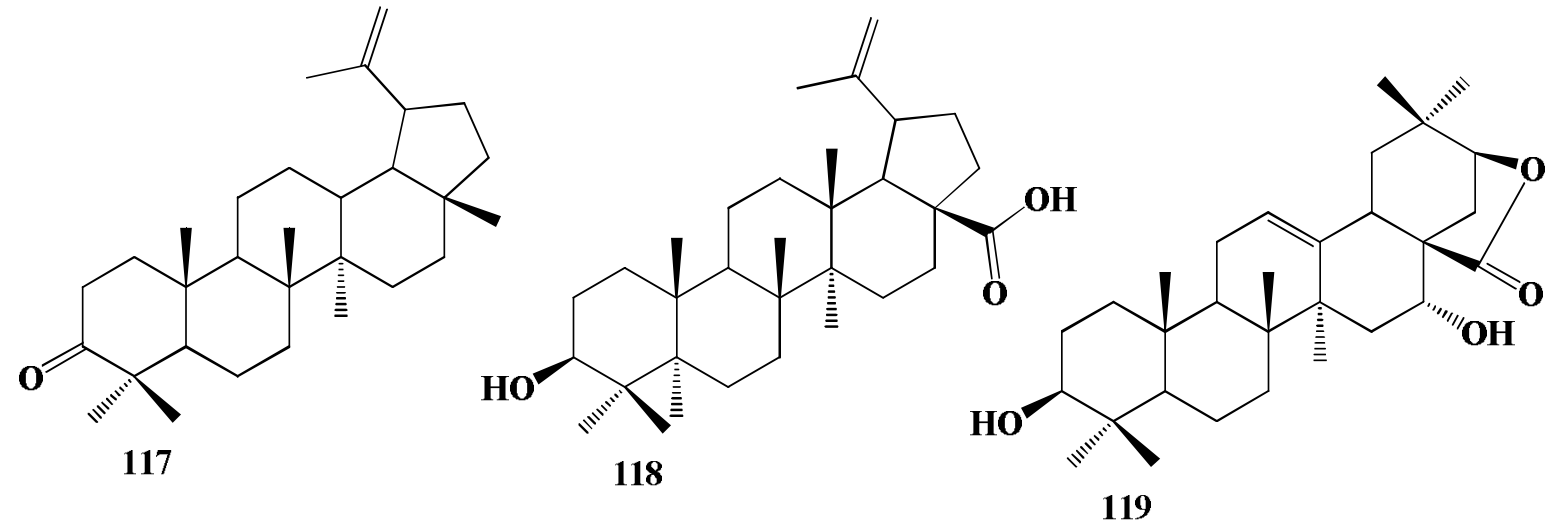<smiles>Oc1cc(O)c2c(c1)O[C@H](c1ccc(O)c(O)c1)C(O)C2</smiles><smiles>OCc1ccccc1</smiles>

Figure 7. Miscellaneous compounds from A. coriaria.

Table 1. Biological properties of the reported phytoconstituents.

\begin{tabular}{cll}
\hline Molecules & \multicolumn{1}{c}{ Biological properties } & \multicolumn{1}{c}{ Ref. } \\
\hline $1-24$ & Antiplasmodial and DPPH free radical scavenging & Yenesewet al., 2012, 1998, 2003 \\
$25-34$ & DPPH free radical scavenging & Bedane et al., 2017 \\
$34-57$ & Cytotoxic & Kader et al., 2001; Runyoroet al., 2015; \\
& & Zhanget al., 2011; Carpaniet al., 1989; \\
& & Pertuitet al., 2017 \\
58 & Anti-trichomonal & Vieiraet al., 2017 \\
$69-87$ & Inhibitory effect against nitric oxide & Fang et al. 2017 \\
$88-93$ & Cytotoxic & Fotso et al., 2017 \\
$94-106$ & Anticandida and cytotoxic & Runyoroet al., 2013; Noteet al., 2016 \\
$107-110$ & Inhibitory effect & Note et al., 2015 \\
$111-113$ & Pro-apoptotic activity (Cytotoxic) & Simo et al., 2017 \\
$114-116$ & Inhibitory activity & Krief et al., 2005 \\
$117-121$ & Antimicrobial & Byamukama et al., 2015 \\
\hline
\end{tabular}




\section{Conclusion}

The molecules from nine medicinal plants have been reviewed. Structurally distinctive different compounds were obtained from these plants. Our study showed that medicinal plants can be a principle source of phytoconstituents as well as medicinal moieties.

\section{References}

Hussain, M.M., Mughal, M.M.R., Alam, M.M., Dastagir, M.G., Billah, A.H.M.M. and Ismail, M. 2010. Antimicrobial activity of n-hexane and ethyl acetate extracts of Erythrina stricta Roxb. Bangladesh J. Microbiol. 27, 65-66.

Hussain, M.M., Tuhin, M.T.H., Akter, F. and Rashid, M.A. 2016a. Constituents of Erythrina- a potential source of secondary metabolites: A review. Bangladesh Pharm. J. 19, 237-253.

Hussain,M.M., Dastagir, M.G., Billah, A.H.M M. And Ismail, M. 2011. Alpinum isoflavone from Erythrina stricta Roxb. Bol. Latinoam. Caribe Plant. Med. Aromat.10, 88-90.

Hussain, M.M., Tahia, F. and Rashid, M.A. $2016 \mathrm{~b}$. Secondary metabolites from some species of Albizzia: A review. Bangladesh Pharm. J. 19, 1-8.

Hussain, M.M., Rahman, M.S., Jabbar, A. and Rashid, M.A. 2008. Phytochemical and biological investigations of Albizzia lebbeck Benth. Bol. Latinoam. Caribe Plant. Med. Aromat. 7, 273-278.

Yenesew, A., Akala, H.M., Twinomuhwezi, H., Chepkirui, C., Irungu, B.N., Eyase, F.L., Mugisha, M.K., Kiremire, B.T., Johnson, J.D. and Waters, N.C. 2012. The antiplasmodial and radical scavenging activities of flavonoids of Erythrina burttii. Acta Tropica 123, 123-127.

Yenesew, A., Midiwo, J.O., Miessner, M., Heydenreich, M. and Peter, M.G. 1998. Two prenylated flavanones from stem bark of Erythrina burttii. Phytochemistry 48, 1439-1443.

Yenesew, A., Irungu, B., Derese, S., Midiwo, J.O., Heydenreich, M. and Peter, M.G. 2003. Two prenylated flavonoids from the stem bark of Erythrina burttii. Phytochemistry63, 445-448.

Bedane, K.G., Kusari, S., Bullach, A., Masesane, I.B., Mihigo, S.O., Spiteller, M. and Majida, R.R.T. 2017. Chemical constituents of the root bark of Erythrina droogmansiana. Phytochem. Lett.20, 84-88.
Kader, M.A., Hoch, J., Berger, J.M., Evans, R., Miller, J.S., Wisse, J.H., Mamber, S.W., Dalton, J.M. and Kingston, D.G.I. 2001. J. Nat. Prod. 64, 536-539.

Runyoro, D.K.B., Joseph, C.C., Ngassapa, O.D., Darokar, M.P., Srivastava, S.K., Matee, M.I.N. and Wright, C.W. 2015. Anticandida agents from a Tanzanian plant Albizzia anthelmintica. J. Chin. Chem. Soc. 62, $1-6$.

Zhang, H., Samadi, A.K., Rao, K.V., Cohen, M.S. and Timmermann, B.N. 2011. Cytotoxic oleanane type saponins from Albizia inundata. J. Nat. Prod .74, 477-482.

Carpani, G., Orsina, F., Sisti, M. and Verotta, L. 1989. Saponins from Albizzia anthelmintica. Phytochemistry 28, 863-866.

Pertuit, D., Larshini, M., Brahim, M.A., Markouk, M., MOffer, A-C., Paululat, T., Delemasure, S., Dutartre, P. and L-Dubois, M.A. 2017. Triterpenoid saponins from the root of Spergularia marginata. Phytochemistry 139, 81-87.

Vieira, P.D.B., Silva, N.L.F., Menezes, C.B., Silva, M.V.D., Silva, D.B., Lopes, N.P., Macedo, A.J., Bastida, J. andTasca, T. 2017. Trichomonicidal and parasite membrane damaging activity of bidesmosic saponins from Manilkara rufula. PLOS ONE12, $\mathrm{e} 0188531$.

Massarani, S.M.A., Gamal, A.A.E., Halim, M.F.A.E., Said, M.S.A., Kader, M.S.A.,Basudan, O.A. and Alqasoumi, S.I. 2017. New acyclic secondary metabolites from the biologically active fraction ofAlbizzia lebbeck. Saudi Pharm. J. 25, 110-121.

Fang, X., Xu, X-K., Wang, G-W., Zeng, R-T., Tian, X-H., Shi, Z-R., Zhou, Z-G., Shen, Y.H. and Zhang, W-D. 2017. Guaianolide sesquiterpenoids from Ainsliaea yunnanesis. Phytochemistry 139, 47-55

Fotso, G.W., Kamga, J., Ngameni, B., Uesugi, S., Ohno, M., Kimura, K-I., Momma, H., Kwon, E., Furuno, H., Shiono, Y., Ingrid, S.K., Yeboah, S.O. and Ngadjui, B.T. 2017. Secondary metabolites with antiproliferative effects from Albizzia glaberrima var glabrescens Oliv. (Mimosoideae). Nat. Product Res.DOI: $\quad$ http://dx.doi.org/10.1080/ $\underline{14786419.2016 .1269097}$.

Runyoro, D.K.B., Srivastava, S.K., Darokar, M.P., Olipa, N.D., Joseph, C.C. and Matee, M.I.N. 2013. Anticandidiasis agents from a Tanzanian plant, Combretum zeyheri. Med. Chem. Res.22, 1258-1262. 
Note, O.P., Azouaou, S.A., Simo, L., Antheaume, C., Guillaume, D., Pegnyemb, D.E., Muller, C.D. and Lobstein, A. 2016. Phenotype-specific apoptosis induced by three triterpenoid saponins from Albizzia glaberrima (Schumach \& Thonn.) Benth. Fitoterapia 109, 80-86.

Note, O.V., Jihu, D., Antheaume, C., Guillaume, D., Pegnyemb, D. E., Kilhoffer, M. C. and Lobstein, A. 2015. Triterpenoid saponins from Albizia boromoensis Aubrev. \& Pellegr. Phytochemistry Lett. 11, 37-42.

Simo, L.M., Note, O.P., Mbing, J.N., Aouazou, S.A., Guillaume, D., Muller, C.D., Pegnyemb, D.E. and A. Lobstein. 2017. New cytotoxic triterpenoid saponins from the roots of Albizzia gummifera. Chem. Biodiversity 14, e1700260.
Krief, S., Thoison, O., Sevenet, T., Wrangham, R.W. and C. Lavaud. 2005. Triterpenoid saponins anthranilates from Albizzia grandibracteata leaves ingested by promates in Uganda. J. Nat. Prod. 68, 897-903

Byamukama,R.,Barbara, G., Namukobe, J., Heydenreich, M. and B. Kiremire. 2015. Bioactive compounds in the stem bark of Albizzia coriaria. Int. J. Bio. Chem. Sci. 9, 1013-1024. 